\title{
Three-Dimensional Morphometric Analysis of Penetrative Depth and Size of Nonarthritic and Degenerative Arthritic Glenoids: Implications for Glenoid Replacement in Shoulder Arthroplasty
}

\author{
Hong Jun Jung, MD, Tae-Seok Nam, MD, Daewon Park, MD, In-Ho Jeon, MD* \\ Department of Orthopedic Surgery, Sahmyook Medical Center, Seoul, \\ *Department of Orthopaedic Surgery, Asan Medical Center, University of Ulsan College of Medicine, Seoul, Korea
}

Background: Shoulder arthroplasty is technically demanding and relies heavily on the precision of surgical techniques. Proper glenoid component sizing plays a crucial role in successful shoulder arthroplasty. We measured the size and penetrative depth of the glenoid for peg or screw fixation in nonarthritic and degenerative arthritic shoulders by using three-dimensional computed tomography to determine the reference dimensions of the glenoid in nonarthritic and degenerative arthritic shoulders.

Methods: From January 2010 to January 2011, data on two groups of patients were collected and reviewed. Group 1 comprised 38 patients who underwent surgical treatment due to fracture of the proximal humerus and who had no evidence of a pathological glenoid. Group 2 comprised 14 patients who underwent surgical treatment due to osteoarthritis of the glenohumeral joint. The height (maximal superoinferior diameter) of the glenoid was measured, and the width (anteroposterior [AP] diameter) of the glenoid was measured at five different levels $(\mathrm{H} 1-\mathrm{H} 5)$. Axial images were taken at $\mathrm{H1} 1-\mathrm{H} 5$ levels, the AP glenoid diameter of each was divided into eight areas, and division points were labeled as W1-W7. The penetrative depths between the near cortex and far cortex of the glenoid (thickness) at each point (W1-W7) were measured.

Results: The overall mean glenoid height was $37.67 \pm 4.09 \mathrm{~mm}$ in nonarthritic glenoids and $39.42 \pm 3.54 \mathrm{~mm}$ in degenerative arthritic glenoids. The nonarthritic glenoid was significantly thicker than the degenerative arthritic glenoid at the H1W3, H1W4, H1W5, H2W7, H3W1, H3W6, H3W7, H4W5, H4W6, H4W7, H5W4, H5W5, H5W6, and H5W7 points. The posteroinferior quadrant had the smallest penetrative depth in both nonarthritic and degenerative arthritic glenoids. Also, the degenerative arthritic glenoids were significantly thinner than the nonarthritic glenoids along the posterior and inferior parts of the glenoid.

Conclusions: The posterior and inferior parts of the degenerative arthritic glenoid appears thinner than the nonarthritic glenoid. Thus, caution has to be taken when drilling the screw hole or inserting screws into the posteroinferior parts, where the glenoid is thinner than $15 \mathrm{~mm}$ on average, to avoid penetration of the far cortex.

Keywords: Glenoid, Penetrative depth, Degenerative arthritis, Shoulder arthroplasty, Three-dimensional computed tomography

Received June 7, 2019; Accepted July 25, 2019

Correspondence to: In-Ho Jeon, MD

Department of Orthopaedic Surgery, Asan Medical Center, University of Ulsan College of Medicine, 88 Olympic-ro 43-gil, Songpa-gu, Seoul 05505, Korea

Tel: +82-2-3010-3896, Fax: +82-2-488-7877

E-mail: jeonchoi@gmail.com
Accurate preoperative planning for shoulder arthroplasty, including exact sizing, is required for good long-term prognosis and function. ${ }^{1)}$ Successful shoulder arthroplasty requires stable initial fixation of the prostheses and a compatible fit between the glenoid component and the underlying glenoid bone. Thus, shoulder arthroplasty is technically demanding and relies heavily on precise preoperative planning and surgical techniques. The glenoid 
Jung et al. Penetrative Depth and Size of Nonarthritic vs. Degenerative Arthritic Glenoid

Clinics in Orthopedic Surgery • Vol. 12, No. 2, $2020 \bullet$ www.ecios.org

is relatively deficient in proper bone stock and weak in strength, making a stable fixation of glenoid components difficult. Proper glenoid component sizing, positioning, and stable fixation all play crucial roles in the success of this operation in terms of favorable long-term prognosis and function. ${ }^{2-5)}$ Despite current efforts to improve the glenoid implant design used in shoulder arthroplasty, glenoid loosening comprises one-third of all reported complications and is the most common indication for revision surgery after shoulder arthroplasty. ${ }^{5-8)}$

Glenoid bone loss commonly occurs in patients with severe osteoarthritis and rotator cuff-deficient arthritis, making proper positioning and stable fixation of the glenoid component difficult. ${ }^{6}$ ) The mismatch between the glenoid and glenoid component can often lead to instability, early loosening, and poor performance of shoulder implants. ${ }^{9)}$ Glenoid component loosening is a major cause of total shoulder arthroplasty failure; ${ }^{8,10-13)}$ some authors report a revision rate of $18.6 \%$ over 20 years of followup. ${ }^{14)}$

Various aspects of the glenoid surface morphology need to be considered for proper prosthetic replacement. The glenoid has been described as pear-shaped; the lower half is larger than the upper half, with a ratio of $1: 0.80 \pm$ $0.01{ }^{15)}$ Numerous recent studies of the size of the glenoid dealt with anteroposterior (AP) and superoinferior diameters. However, little attention has been paid to the penetrative depth of the glenoid. ${ }^{3,9,16,17)}$
In this study, we measured the size and penetrative depth of the glenoid in nonarthritic and degenerative arthritic shoulders by using three-dimensional computed tomography (3D CT). The regional variations of each parameter were analyzed and the results were compared between nonarthritic and degenerative arthritic shoulders. These data will be useful in the design of glenoid components and preoperative templating.

\section{METHODS}

The protocol of this study was reviewed and approved by the Institutional Review Board of the Asan Medical Center (IRB No. AMC-IRB 2019-0684). The informed consent was waived.

\section{Patient Enrollement}

From January 2010 to January 2011, data on two groups of patients were collected and reviewed. Group 1 comprised 38 patients who underwent surgical treatment due to fracture of the proximal humerus. Among these patients, there was no radiographic evidence of osteoarthritis, rotator cuff-deficient arthritis, or inflammatory arthropathy of the glenohumeral joint. The morphometric data from the 38 patients were derived from our prior study. ${ }^{18)}$ Group 2 comprised 14 patients who underwent surgical treatment due to osteoarthritis of the glenohumeral joint. All CT scans were obtained before surgical treatment. Group
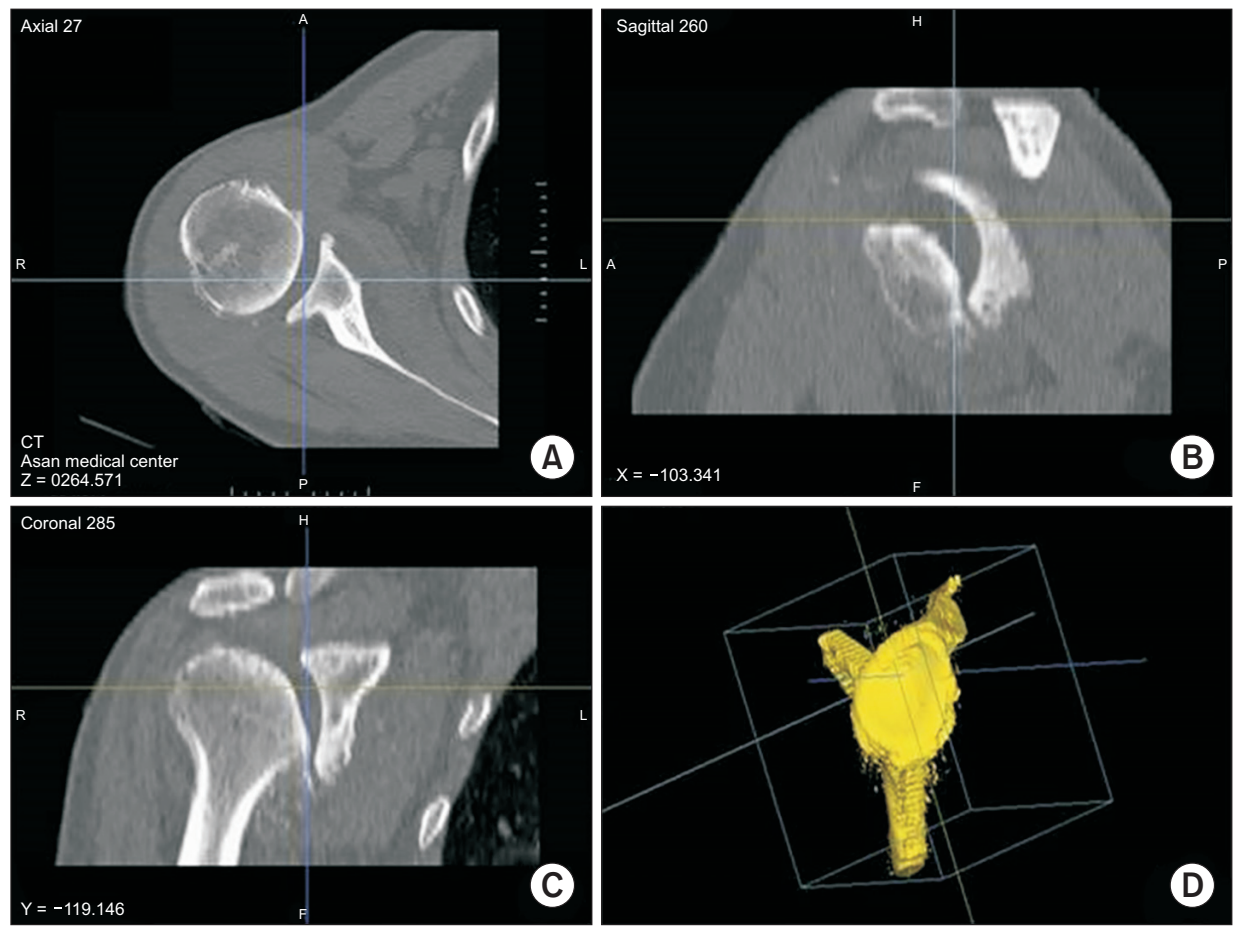

Fig. 1. Axial (A), sagittal (B), and coronal (C) computed tomographic images captured during the investigation using the V-works program. (D) Three-dimensional reconstructed image of the glenoid. Any plane of the glenoid can be seen by simple operation. It shows the articular surface of the glenoid. Reproduced from Jung et al. Clin Anat. 2012;25(8):1043-50. ${ }^{18)}$ 
Jung et al. Penetrative Depth and Size of Nonarthritic vs. Degenerative Arthritic Glenoid

Clinics in Orthopedic Surgery • Vol. 12, No. 2, $2020 \bullet$ www.ecios.org

1 consisted of 17 men and 21 women with a mean age of 61.9 years ( $61.9 \pm 12.1$ years; range, $40-71$ years), and group 2 consisted of two men and 12 women with a mean age of 70.4 years ( $70.4 \pm 15.4$ years; range, $45-81$ years).

\section{CT Imaging and Software}

The analysis methods used were identical to those in our previous study. ${ }^{18)} \mathrm{CT}$ image cuts of $0.2 \mathrm{~mm}$ thickness were

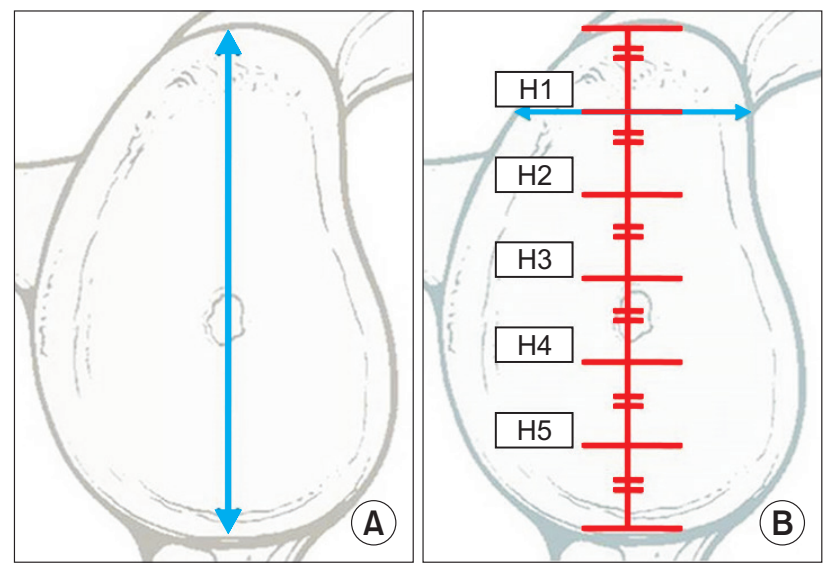

Fig. 2. Schematic illustration for measurements of the height $(A)$ and width $(B)$ of the glenoid. Reproduced from Jung et al. Clin Anat. 2012;25(8):1043-50. ${ }^{18)}$ taken using a multidetector Somatom Sensation 16 (Siemens, Forchheim, Germany). V-works software ver. 4.0001 (3D Medical Imaging Software, Cybermed Inc., Seoul, Korea) was used to reconstruct all the CT images with bone density into 3D images with unnecessary areas eliminated through humeral head subtraction and imaging artifacts removed.

\section{Height and Width Measurement}

Reconstructed images were rotated $360^{\circ}$ to obtain a perfect en face view of the glenoid, so that the superoinferior and AP diameters of the glenoid could be measured irrespective of its anteversion or inclination (Fig 1). The height (maximal superoinferior diameter) of the glenoid was measured, and the width (AP diameter) was measured at five different levels (H1-H5) (Fig. 2).

\section{Penetrative Depth Measurement}

Axial images were taken at $\mathrm{H} 1-\mathrm{H} 5$ levels, with the AP glenoid diameter of each divided into eight areas (division points are labeled as W1-W7). The penetrative depths between the near cortex and far cortex of the glenoid (thickness) at each point (W1-W7) were measured (Fig. 3). All measurements were conducted twice by one researcher (HJJ; orthopaedic surgeon, shoulder specialist) and were averaged.
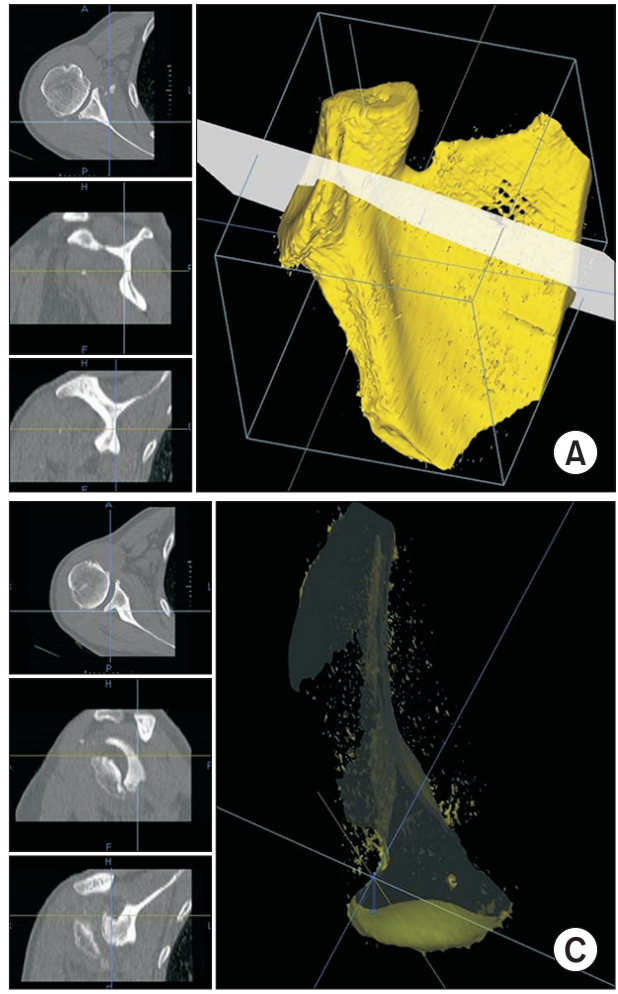
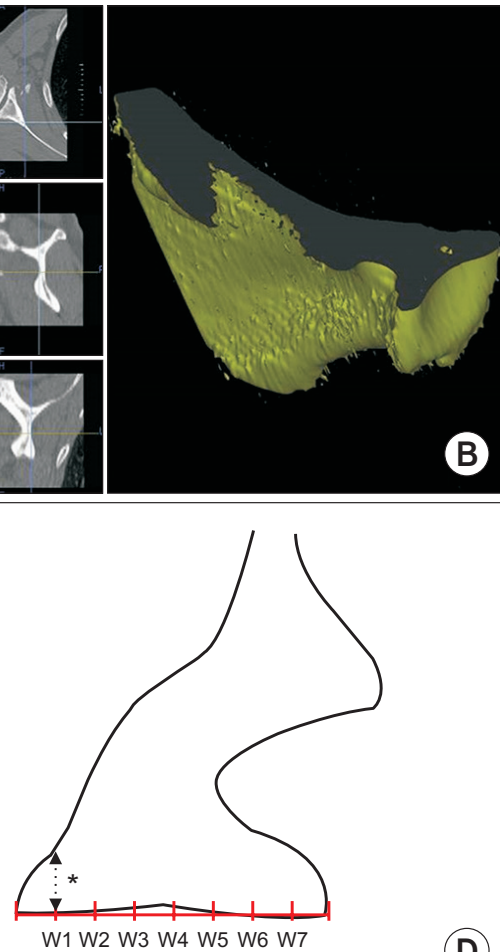

(D)
Fig. 3. Measurement of the glenoid penetration depth. (A-C) True axial images of the glenoid were obtained by threedimensional image reconstruction. (D) Schematic illustration of the true axial image of the glenoid. The distance (asterisk) between the proximal cortex of the articular surface and the far cortex was measured at seven points from the anterior to the posterior margin of the glenoid in six axial sections. Reproduced from Jung et al. Clin Anat. 2012;25(8): $1043-50^{18)}$ 
Jung et al. Penetrative Depth and Size of Nonarthritic vs. Degenerative Arthritic Glenoid

Clinics in Orthopedic Surgery • Vol. 12, No. 2, $2020 \bullet$ www.ecios.org

\section{Statistical Analysis}

All statistical analyses were performed using SPSS ver. 18.0 (SPSS Inc., Chicago, IL, USA). Differences in the glenoid width, height, and penetrative depth were compared between nonarthritic and degenerative arthritic glenoids by using independent $t$-tests, with $p<0.05$ considered statistically significant.

\section{RESULTS}

Group 1 (nonarthritic glenoids) consisted of 17 men and 21 women with a mean age of 61.9 years $(61.9 \pm 12.1$ years; range, 40-71 years), and group 2 (degenerative arthritic glenoids) consisted of two men and 12 women with a mean age of 70.4 years ( $70.4 \pm 15.4$ years; range, $45-81$ years). The overall mean glenoid height was $37.67 \pm 4.09$ $\mathrm{mm}$ in nonarthritic glenoids and $39.42 \pm 3.54 \mathrm{~mm}$ in degenerative arthritic glenoids $(p=0.132)$.

The glenoid was narrowest at the superior $1 / 5$ point and widest at the inferior $4 / 5$ point in both nonarthritic and degenerative arthritic glenoids. The width of nonarthritic glenoids increased gradually from a mean of 16.42 $\pm 2.18 \mathrm{~mm}$ at the $\mathrm{H1}$ level to a mean of $27.52 \pm 2.93 \mathrm{~mm}$ at the $\mathrm{H} 4$ level and slightly decreased thereafter to 22.96 $\pm 2.80 \mathrm{~mm}$ at the $\mathrm{H} 5$ level. The width of degenerative arthritic glenoids also increased gradually from a mean of $19.43 \pm 2.87 \mathrm{~mm}$ at the $\mathrm{H} 1$ level to a mean of $28.68 \pm 3.30$ $\mathrm{mm}$ at the $\mathrm{H} 4$ level and slightly decreased thereafter to $24.29 \pm 3.18 \mathrm{~mm}$ at the $\mathrm{H} 5$ level. At the $\mathrm{H} 1, \mathrm{H} 2$, and $\mathrm{H} 5$ levels, the width was significantly greater in the degenerative arthritic glenoids than in the nonarthritic glenoids $(p<0.05)$ (Table 1).

The penetrative depth (thickness) of the nonarthritic glenoids increased as the reference point, which was at

\begin{tabular}{|c|c|c|c|}
\hline Level & Group $1(n=38)$ & Group $2(n=14)$ & $p$-value \\
\hline Height (mm) & $37.67 \pm 4.09$ & $39.42 \pm 3.54$ & 0.132 \\
\hline \multicolumn{4}{|l|}{ Width (mm) } \\
\hline $\mathrm{H} 1$ & $16.42 \pm 2.18$ & $19.43 \pm 2.87$ & $<0.05$ \\
\hline $\mathrm{H} 2$ & $21.94 \pm 2.82$ & $24.76 \pm 3.49$ & $<0.05$ \\
\hline $\mathrm{H} 3$ & $26.89 \pm 3.20$ & $28.13 \pm 3.19$ & 0.187 \\
\hline $\mathrm{H} 4$ & $27.52 \pm 2.93$ & $28.69 \pm 3.31$ & 0.187 \\
\hline H5 & $22.96 \pm 2.80$ & $24.29 \pm 3.18$ & $<0.05$ \\
\hline
\end{tabular}

Values are presented as mean \pm standard deviation.
Table 2. The Penetrative Depth of the Nonarthritic and Degenerative Arthritic Glenoids in Enrolled Patients

\begin{tabular}{|c|c|c|c|c|}
\hline \multirow{2}{*}{ Level } & \multirow{2}{*}{ Point } & \multicolumn{3}{|c|}{ Penetration depth } \\
\hline & & Group $1(n=38)$ & Group $2(n=14)$ & $p$-value \\
\hline \multirow[t]{7}{*}{$\mathrm{H} 1$} & W1 & $14.90 \pm 2.44$ & $13.94 \pm 4.09$ & \\
\hline & W2 & $22.09 \pm 4.88$ & $18.51 \pm 4.58$ & \\
\hline & W3 & $25.71 \pm 4.57$ & $21.22 \pm 3.10$ & $<0.05$ \\
\hline & W4 & $28.47 \pm 3.18$ & $21.17 \pm 4.04$ & $<0.05$ \\
\hline & W5 & $24.29 \pm 3.68$ & $17.70 \pm 4.58$ & $<0.05$ \\
\hline & W6 & $15.94 \pm 3.97$ & $12.44 \pm 3.83$ & \\
\hline & W7 & $10.15 \pm 3.86$ & $7.92 \pm 2.71$ & \\
\hline \multirow[t]{7}{*}{$\mathrm{H} 2$} & W1 & $9.36 \pm 5.23$ & $11.02 \pm 3.81$ & \\
\hline & W2 & $17.40 \pm 5.33$ & $17.34 \pm 6.02$ & \\
\hline & W3 & $23.45 \pm 4.72$ & $23.20 \pm 5.54$ & \\
\hline & W4 & $28.43 \pm 5.45$ & $27.65 \pm 4.37$ & \\
\hline & W5 & $30.51 \pm 4.63$ & $27.59 \pm 6.43$ & \\
\hline & W6 & $11.88 \pm 5.86$ & $9.43 \pm 2.18$ & \\
\hline & W7 & $7.45 \pm 1.86$ & $6.14 \pm 1.57$ & $<0.05$ \\
\hline \multirow[t]{7}{*}{ H3 } & W1 & $6.72 \pm 1.52$ & $5.77 \pm 1.36$ & $<0.05$ \\
\hline & W2 & $14.21 \pm 3.67$ & $12.95 \pm 3.67$ & \\
\hline & W3 & $21.34 \pm 4.11$ & $21.81 \pm 4.01$ & \\
\hline & W4 & $27.04 \pm 5.47$ & $29.15 \pm 4.47$ & \\
\hline & W5 & $34.38 \pm 4.29$ & $32.90 \pm 4.79$ & \\
\hline & W6 & $14.18 \pm 4.31$ & $8.08 \pm 3.42$ & $<0.05$ \\
\hline & W7 & $6.92 \pm 1.94$ & $5.64 \pm 2.34$ & $<0.05$ \\
\hline \multirow[t]{7}{*}{$\mathrm{H} 4$} & W1 & $7.99 \pm 1.94$ & $7.24 \pm 1.99$ & \\
\hline & W2 & $14.31 \pm 3.10$ & $12.92 \pm 2.87$ & \\
\hline & W3 & $21.81 \pm 5.05$ & $21.09 \pm 4.61$ & \\
\hline & W4 & $27.10 \pm 4.03$ & $26.66 \pm 3.08$ & \\
\hline & W5 & $33.74 \pm 6.51$ & $26.15 \pm 3.48$ & $<0.05$ \\
\hline & W6 & $17.61 \pm 3.83$ & $9.55 \pm 2.79$ & $<0.05$ \\
\hline & W7 & $8.24 \pm 1.52$ & $6.76 \pm 1.29$ & $<0.05$ \\
\hline \multirow[t]{7}{*}{ H5 } & W1 & $9.92 \pm 4.13$ & $10.32 \pm 3.88$ & \\
\hline & W2 & $19.81 \pm 6.31$ & $18.05 \pm 6.14$ & \\
\hline & W3 & $26.55 \pm 5.53$ & $24.49 \pm 3.49$ & \\
\hline & W4 & $31.21 \pm 5.88$ & $26.49 \pm 3.68$ & $<0.05$ \\
\hline & W5 & $30.25 \pm 5.64$ & $21.82 \pm 4.37$ & $<0.05$ \\
\hline & W6 & $19.28 \pm 4.86$ & $12.80 \pm 3.84$ & $<0.05$ \\
\hline & W7 & $11.08 \pm 3.77$ & $8.13 \pm 3.31$ & $<0.05$ \\
\hline
\end{tabular}

Values are presented as mean \pm standard deviation. 
Jung et al. Penetrative Depth and Size of Nonarthritic vs. Degenerative Arthritic Glenoid

Clinics in Orthopedic Surgery • Vol. 12, No. 2, $2020 \bullet$ www.ecios.org

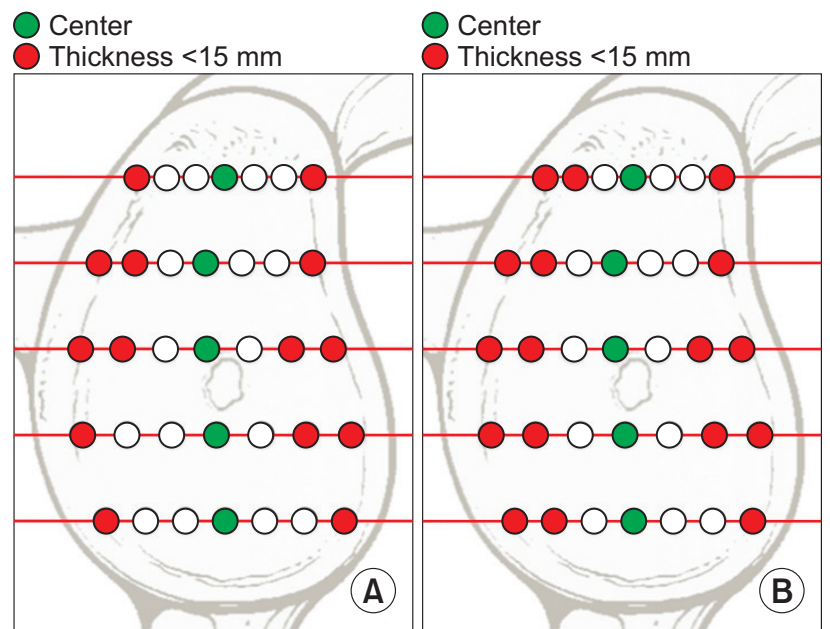

Fig. 4. The penetration depth of the glenoid in nonarthritic $(A)$ and degenerative arthritic (B) glenoids.

the $5 / 7$ point from the anterior margin and progressed in the posterior direction. The greatest measurement was at the W5 point at the $\mathrm{H} 3$ level, and also the $\mathrm{W} 5$ point was the greatest at all the other levels. The penetrative depth of the degenerative arthritic glenoids increased as the reference point, which was at the $4 / 7$ point from the anterior margin, progressed in the posterior direction. The greatest measurement was at the W5 point at the $\mathrm{H} 3$ level, but the W4 point was the greatest at all the other levels (Table 2).

The glenoid depth was significantly greater $(p<0.05)$ in nonarthritic glenoids than in degenerative arthritic glenoids at the H1W3, H1W4, H1W5, H2W7, H3W1, H3W6, H3W7, H4W5, H4W6, H4W7, H5W4, H5W5, H5W6, and H5W7 points (Figs. 4 and 5).

\section{DISCUSSION}

We demonstrated regional variations in glenoid penetrative depth in nonarthritic and degenerative arthritic glenoids and a significant difference in size and penetrative depth between the nonarthritic and degenerative arthritic glenoids. The posteroinferior quadrant had the smallest distance from the glenoid articular cartilage to the far cortex in both nonarthritic and degenerative arthritic glenoids, which implies that this region is at the greatest risk of screw penetration and possible cement leakage. The potential complications of penetrating the far cortex of the glenoid with the screws are leakage of cement, stable glenoid component fixation, early loosening, and neurovascular damage. Also, we found that the degenerative arthritic glenoids were significantly thinner than the nonarthritic glenoids along the posterior and inferior parts of

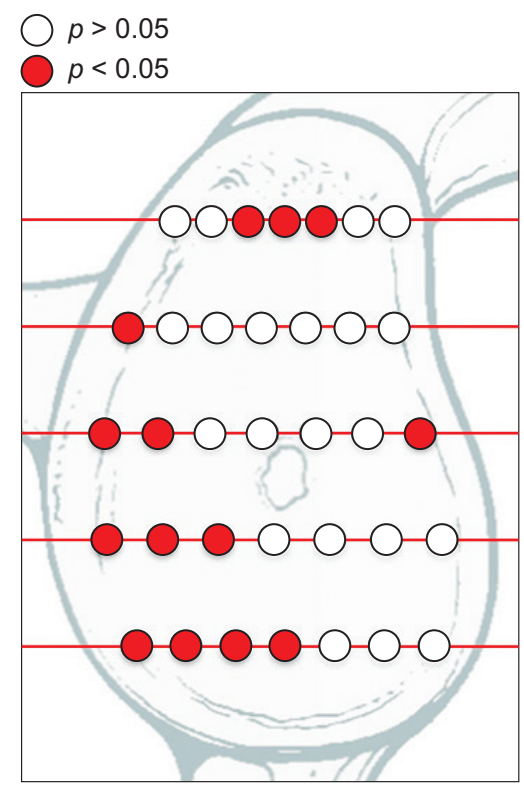

Fig. 5. The penetration depth of the glenoid in different areas in nonarthritic and degenerative arthritic glenoids. The black dots indicate areas of statistically significant differences between nonarthritic and degenerative arthritic glenoids.

the glenoid.

In the degenerative arthritic glenoids, there were multiple locations where the glenoid was thinner than that in their nonarthritic counterparts, and the thickness was less than $15 \mathrm{~mm}$. As the far cortex is concentrated at the posteroinferior area of the glenoid, care should be taken not to penetrate the structure during drilling in this area. Thus, on the basis of these findings, when the glenoid component is modified, the glenoid height, width, and penetrative depth should be considered. In addition, glenoid peg length should take account of degenerative arthritic glenoid penetrative depth, especially in the posteroinferior quadrant.

Previous studies have not reported the glenoid penetrative depth because most studies have simply measured the diameter of the glenoid in cadavers with CT and magnetic resonance imaging (MRI) to provide the dimensional data. ${ }^{3,16,17,19)}$ In our study, we measured the height and width in the AP view and the penetrative depth in the axial view and then constructed the glenoid in $3 \mathrm{D}$. In one comparative study of two-dimensional (2D) and 3D CT images in 12 cadaveric scapulae, the authors indicated that the 3D CT images were more reliable as they better reflected the true anatomy. ${ }^{19)}$ We also found $3 \mathrm{D}$ reconstruction $\mathrm{CT}$ images to be a useful tool during preoperative evaluation in the clinical setting. Thus, we used 3D CT images in this study to measure the glenoid surface and vault and the 
Jung et al. Penetrative Depth and Size of Nonarthritic vs. Degenerative Arthritic Glenoid

Clinics in Orthopedic Surgery • Vol. 12, No. 2, $2020 \bullet$ www.ecios.org

penetrative depth in various anatomical regions.

Iannotti et al. ${ }^{15)}$ conducted a study of the length and width of the glenoid by using 2D and 3D CT and MRI. In the anatomical study of 140 glenohumeral joints, they reported that the shape of the glenoid vault is pear-shaped with a mean maximal height of $39 \mathrm{~mm}$ (range, 30-48 $\mathrm{mm}$ ), with an average upper AP width of $23 \mathrm{~mm}$ (range, 18-30 mm), and an average lower AP width of $29 \mathrm{~mm}$ (range, 21-35 mm). In another study, De Wilde et al. ${ }^{20)}$ measured glenoids obtained from 98 cadaver shoulders and reported that the maximum height was $40.1 \mathrm{~mm}$ on average and the lower-half width was $29.1 \mathrm{~mm}$ on average. Similarly, we found the lower half was larger than the top half for both nonarthritic and degenerative arthritic glenoids. However, the height and width, especially the upper-half width, of the degenerative arthritic glenoids were slightly larger than those of the nonarthritic glenoids. These findings are thought to have been caused by changes in glenoid morphology, subchondral bone change, increased bone-to-bone contact area, and bony spur formation associated with degenerative arthritic glenoids. These results indicate that the degenerative arthritic glenoid is more round in shape compared to the pear-shaped nonarthritic glenoid described in Iannotti et al.'s study. ${ }^{15}$

In a study on the thickness of the glenoid, Codsi et al. ${ }^{17)}$ used CT scans of 69 scapulae to make a triangular simplified axial cut model and a model of the horizontal cross section of the glenoid of the joint and the region, and measured the depths from the sides corresponding to the joint in five horizontal sections, which were $28.5 \mathrm{~mm}$, $19.75 \mathrm{~mm}, 18.0 \mathrm{~mm}, 20.75 \mathrm{~mm}$, and $22.5 \mathrm{~mm}$. In addition, Codsi et al. ${ }^{3)}$ divided the joints into upper, middle, and lower sections by using CT and identified the ideal insertion location of screws and the maximum screw length required in order to avoid the screw passing through the cortex of the glenoid when inserted at an angle of $15^{\circ}$. We focused on understanding the morphology of the glenoid by obtaining detailed data on its actual size, including glenoid penetrative depth in addition to its height and width.

The measurements may have been affected by various factors including the anteversion and inclination of the glenoid and the position of the scapula. To minimize such variation, we conducted the measurement with the $3 \mathrm{D} C \mathrm{CT}$ images using a standard protocol. Despite the lack of direct comparison, it is likely that there are few, if any, differences in glenoid thickness between Koreans and Westerners. For example, Cabezas et al. ${ }^{21)}$ conducted an analysis of morphologic variability of the shoulder between the populations of North American and East Asia. They reported that the East Asian population exhibited smaller shoulder morphometrics than did the North American cohort, especially regarding the glenoid height and inferior glenoid width. They reported that these data could provide some additional factors to consider when choosing an optimal glenoid implant for the East Asian population, in addition to creating future designs that may better accommodate this population.

The glenoid penetrative depth is of clinical signifi-

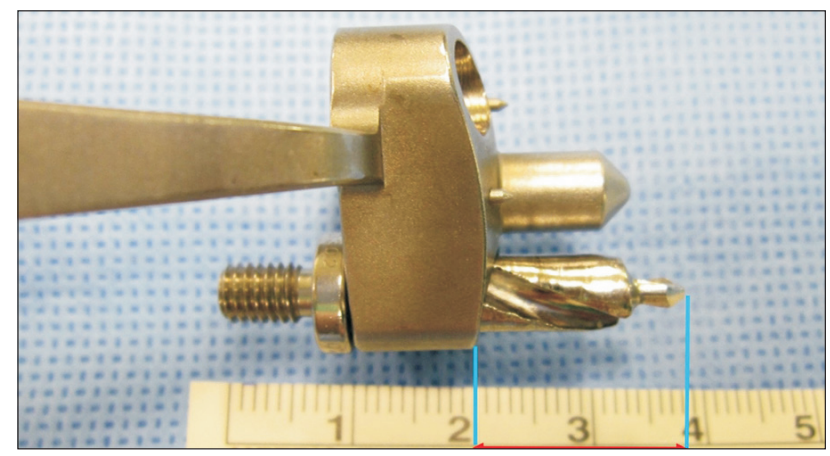

Fig. 7. The jig used for drilling yields a hole with a depth above $15 \mathrm{~mm}$. Reproduced from Jung et al. Clin Anat. 2012;25(8):1043-50. ${ }^{181}$
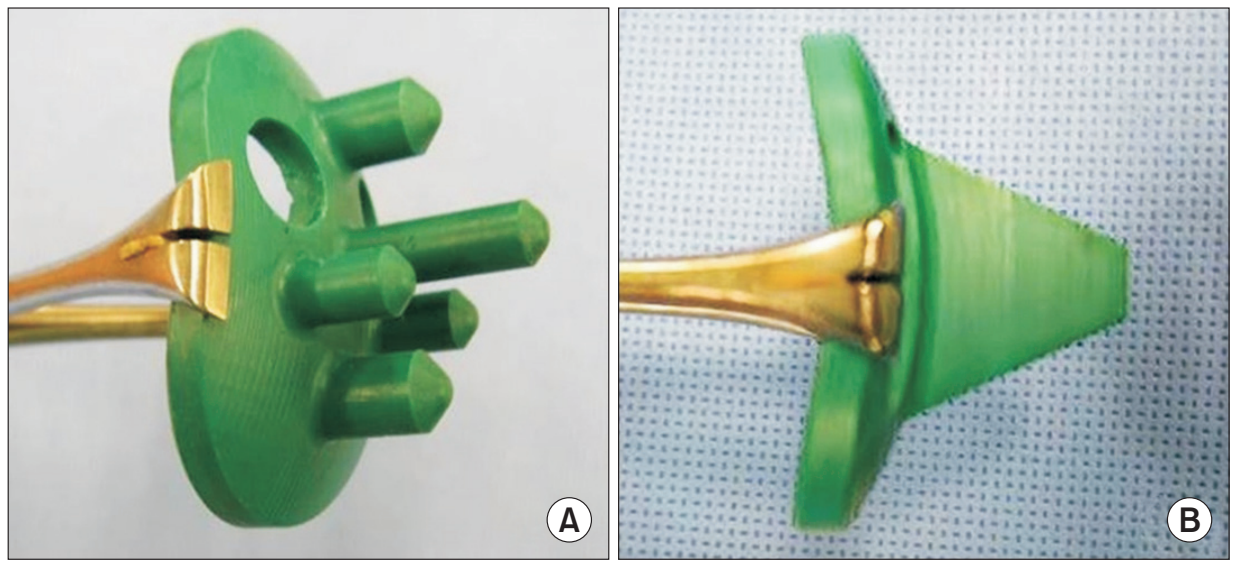

Fig. 6. A sample glenoid trial insert: pegged component (A) and keeled component (B). Reproduced from Jung et al. Clin Anat. 2012;25(8):1043-50. ${ }^{18)}$ 
cance, particularly when placing glenoid components for total shoulder arthroplasty (Fig. 6). ${ }^{8)}$ The use of a fivepegged glenoid component can prevent the rocking horse phenomenon, thus providing more stable fixation. ${ }^{22)}$ Drilling of the glenoid for pegs creates a hole of more than 15 $\mathrm{mm}$ in depth (Fig. 7), which can penetrate the far cortex if a glenoid depth is less than $15 \mathrm{~mm}$, resulting in cement leakage and making stable glenoid component fixation difficult. In addition, complications of penetrating the far cortex of the glenoid with the screws include difficulty in achieving stable glenoid component fixation, early glenoid component loosening, and neurovascular damage.

There are several limitations to this study. First, the sample size was small. Further studies with a larger sample size are needed. Second, we did not perform an interobserver study. This may have affected the reliability and reproducibility of our measurements. Third, the CT scans we used for glenoid size measurement have a possible limitation in that the thickness of the articular cartilage was not included. However, we fixed the glenoid component after removing the glenoid cartilage, and it is the size of the glenoid bone that is crucial. In addition, while the desired outcome of our study was to improve our knowledge of glenoid anatomy and possibly improve glenoid design for total shoulder arthroplasty, we did not include people of all ages; however, the population we selected is most clinically relevant given that the majority of shoulder arthroplasties are conducted in this age group.

In summary, we measured the mean height, width, and penetrative depth (thickness) of nonarthritic and degenerative arthritic glenoids to delineate the glenoid vault as a whole. Information from this study on regional variations in the $3 \mathrm{D}$ glenoid morphology may be useful in determining ideal fixation within the glenoid and in developing new designs for glenoid implants and glenoid implant fixation. This study also showed that the posterior and inferior parts of the glenoid are thinner than the other parts of the glenoid. Thus, caution has to be taken when drilling the screw hole or inserting screws into the posteroinferior parts, where the glenoid is thinner than 15 $\mathrm{mm}$ on average, to avoid penetration of the far cortex. In addition, these anatomical features of the glenoid should be considered in the development of shoulder arthroplasty implants in the future.

\section{CONFLICT OF INTEREST}

No potential conflict of interest relevant to this article was reported.

\section{REFERENCES}

1. Wirth MA, Loredo R, Garcia G, Rockwood CA Jr, Southworth C, Iannotti JP. Total shoulder arthroplasty with an allpolyethylene pegged bone-ingrowth glenoid component: a clinical and radiographic outcome study. J Bone Joint Surg Am. 2012;94(3):260-7.

2. Bohsali KI, Wirth MA, Rockwood CA Jr. Complications of total shoulder arthroplasty. J Bone Joint Surg Am. 2006;88(10):2279-92.

3. Codsi MJ, Bennetts C, Powell K, Iannotti JP. Locations for screw fixation beyond the glenoid vault for fixation of glenoid implants into the scapula: an anatomic study. J Shoulder Elbow Surg. 2007;16(3 Suppl):S84-9.

4. Harman M, Frankle M, Vasey M, Banks S. Initial glenoid component fixation in "reverse" total shoulder arthroplasty: a biomechanical evaluation. J Shoulder Elbow Surg. 2005;14(1 Suppl S):162S-167S.

5. Matsen FA 3rd, Clinton J, Lynch J, Bertelsen A, Richardson ML. Glenoid component failure in total shoulder arthroplasty. J Bone Joint Surg Am. 2008;90(4):885-96.

6. Cofield RH. Total shoulder arthroplasty with the Neer prosthesis. J Bone Joint Surg Am. 1984;66(6):899-906.
7. Sperling JW, Cofield RH, Rowland CM. Minimum fifteenyear follow-up of Neer hemiarthroplasty and total shoulder arthroplasty in patients aged fifty years or younger. J Shoulder Elbow Surg. 2004;13(6):604-13.

8. Strauss EJ, Roche C, Flurin PH, Wright T, Zuckerman JD. The glenoid in shoulder arthroplasty. J Shoulder Elbow Surg. 2009;18(5):819-33.

9. Merrill A, Guzman K, Miller SL. Gender differences in glenoid anatomy: an anatomic study. Surg Radiol Anat. 2009;31(3):183-9.

10. Gregory T, Hansen U, Emery RJ, Augereau B, Amis AA. Developments in shoulder arthroplasty. Proc Inst Mech Eng H. 2007;221(1):87-96.

11. Hoenecke HR Jr, Tibor LM, D'Lima DD. Glenoid morphology rather than version predicts humeral subluxation: a different perspective on the glenoid in total shoulder arthroplasty. J Shoulder Elbow Surg. 2012;21(9):1136-41.

12. Sharma GB, McMahon PJ, Robertson DD. Structure modeling of the glenoid: relevance to shoulder arthroplasty. J Orthop Res. 2014;32(11):1471-8. 
13. Williams GR, Abboud JA. Total shoulder arthroplasty: glenoid component design. J Shoulder Elbow Surg. 2005;14(1 Suppl S):122S-128S.

14. Singh JA, Sperling JW, Cofield RH. Revision surgery following total shoulder arthroplasty: analysis of 2588 shoulders over three decades (1976 to 2008). J Bone Joint Surg Br. 2011;93(11):1513-7.

15. Iannotti JP, Gabriel JP, Schneck SL, Evans BG, Misra S. The normal glenohumeral relationships: an anatomical study of one hundred and forty shoulders. J Bone Joint Surg Am. 1992;74(4):491-500.

16. Churchill RS, Brems JJ, Kotschi H. Glenoid size, inclination, and version: an anatomic study. J Shoulder Elbow Surg. 2001;10(4):327-32.

17. Codsi MJ, Bennetts C, Gordiev K, et al. Normal glenoid vault anatomy and validation of a novel glenoid implant shape. J Shoulder Elbow Surg. 2008;17(3):471-8.
18. Jung HJ, Jeon IH, Ahn TS, et al. Penetration depth and size of the nonarthritic glenoid: implications for glenoid replacement. Clin Anat. 2012;25(8):1043-50.

19. Kwon YW, Powell KA, Yum JK, Brems JJ, Iannotti JP. Use of three-dimensional computed tomography for the analysis of the glenoid anatomy. J Shoulder Elbow Surg. 2005;14(1):8590.

20. De Wilde LF, Berghs BM, Audenaert E, Sys G, Van Maele GO, Barbaix E. About the variability of the shape of the glenoid cavity. Surg Radiol Anat. 2004;26(1):54-9.

21. Cabezas AF, Krebes K, Hussey MM, et al. Morphologic variability of the shoulder between the populations of North American and East Asian. Clin Orthop Surg. 2016;8(3):2807.

22. Orr TE, Carter DR, Schurman DJ. Stress analyses of glenoid component designs. Clin Orthop Relat Res. 1988;(232):21724. 